\title{
Characterizing the nanoacoustic superlattice in a phonon cavity using a piezoelectric single quantum well
}

\author{
Kung-Hsuan Lin and Chieh-Feng Chang \\ Department of Electrical Engineering, National Taiwan University, Taipei 10617, Taiwan \\ and Graduate Institute of Electro-Optical Engineering, National Taiwan University, Taipei 10617, Taiwan
}

Chang-Chi Pan and Jen-Inn Chyi

Optical Sciences Center, National Central University, Chung-Li 32054, Taiwan

Stacia Keller, Umesh Mishra, and Steven P. DenBaars

Department of Electrical and Computer Engineering, University of California, Santa Barbara, California 93106

\author{
Chi-Kuang Sun ${ }^{\text {a) }}$ \\ Department of Electrical Engineering, National Taiwan University, Taipei 10617, Taiwan \\ and Graduate Institute of Electro-Optical Engineering, National Taiwan University, Taipei 10617, Taiwan
}

(Received 13 May 2006; accepted 22 August 2006; published online 2 October 2006)

\begin{abstract}
We have experimentally and theoretically investigated, both in the time domain and in the frequency domain, the characteristics of a nanoacoustic mirror, which is a 17-period $8 \mathrm{~nm} / 8 \mathrm{~nm}$ $\mathrm{Al}_{0.7} \mathrm{Ga}_{0.3} \mathrm{~N} / \mathrm{Al}_{0.2} \mathrm{Ga}_{0.8} \mathrm{~N}$ superlattice and its first phononic band gap frequency is centered at $\sim 280 \mathrm{GHz}$. By using a femtosecond optical pulse to excite and detect the nanoacoustic strain pulses with an $\mathrm{In}_{0.2} \mathrm{Ga}_{0.8} \mathrm{~N}$ single quantum well, we directly measured the transient dynamics of the acoustic nanowaves inside a phonon cavity composed of the studied nanoacoustic mirror. The phase-resolved reflection transfer function of the phononic band gap superlattice and the properties of the nanophononic cavity have been experimentally obtained and investigated. (c) 2006 American Institute of Physics. [DOI: 10.1063/1.2358321]
\end{abstract}

A structure of alternating materials with different elastic properties forms phononic band gaps. ${ }^{1}$ With a nanometer periodicity in a superlattice structure, the phononic band gap frequencies are usually in the subterahertz to terahertz regime and a nanoacoustic superlattice is treated with a zonefolded phonon picture. $^{2}$ Due to the periodic structure, the acoustic phonon branches are folded, resulting in the opening of energy gaps at the center and the boundaries of the artificially formed Brillouin zones. ${ }^{2}$ On the other hand, the transmission or reflection spectra of a finite size nanoacoustic superlattice can be calculated by the transfer-matrix method based on the continuum elastic theory. ${ }^{3}$ The physical properties of the energy stop bands have previously been investigated from the energy point of view by measuring the transmitted acoustic energy with a superconductor bolometer ${ }^{4,5}$ or by Raman scattering. ${ }^{2,6}$ Due to the high band gap frequencies, nanoscale-superlattice structures should be treated as nanoacoustic mirrors to reflect acoustic nanowaves ${ }^{7,8}$ (or nanoacoustic waves 910 ) for the emerging "nanoultrasonics" 11 or "nanophononics." 12 However, there were no experimental studies that directly investigated the band gap phenomena and characterized the nanoacoustic superlattice from the wave point of view in the time domain.

In this letter, we demonstrate a time-resolved method to characterize a nanoacoustic superlattice. The nanoacoustic superlattice under study has a 17-period $8 \mathrm{~nm} / 8 \mathrm{~nm}$ $\mathrm{Al}_{0.7} \mathrm{Ga}_{0.3} \mathrm{~N} / \mathrm{Al}_{0.2} \mathrm{Ga}_{0.8} \mathrm{~N}$ structure, and the theoretical first phononic band gap is from 267 to $291 \mathrm{GHz}$. For such a high acoustic frequency, we utilized a $7 \mathrm{~nm}$ piezoelectric single quantum well (SQW) as a broadband acoustic sensor to di-

\footnotetext{
a) Author to whom correspondence should be addressed; electronic mail:
} sun@cc.ee.ntu.edu.tw rectly measure the acoustic nanowaves propagating inside the nanophononic cavity formed by superlattice mirrors. By analyzing the acoustic nanowave oscillations, the reflection transfer function of the nanoacoustic superlattice around the first phononic band gap with phase information was experimentally obtained.

The detection mechanism of the acoustic nanowaves through a piezoelectric SQW is based on a recent investigation of light transmission through piezoelectric multiple quantum wells modulated by coherent acoustic phonons. ${ }^{7,13,14}$ While coherent acoustic phonons enter the SQW, they modify the resonant condition of photon absorption through piezoelectric and deformation-potential coupling effects. The modulated absorption of the SQW due to coherent acoustic phonons can be macroscopically described with a strain wave and a sensitivity function. ${ }^{13,14}$ By recording the changes of light transmission through the SQW as a function of time delay, we can thus experimentally measure the time-varying strain amplitude in the SQW region.

When a strain wave $w(t-z / V)$ travels through the SQW, where $V$ is the sound velocity, the modulated optical transmission $\Delta T$ can be expressed as

$$
\Delta T(t)=\int_{-\infty}^{\infty} d z w(t-z / V) F\left(z, \nu_{0}\right),
$$

where $F\left(z, \nu_{0}\right)$ is the sensitivity function of the SQW at the optical frequency $\nu_{0} .{ }^{14}$ Here, we neglected the $2.3 \%$ strain reflectivity at the $\operatorname{In}_{0.2} \mathrm{Ga}_{0.8} \mathrm{~N} / \mathrm{GaN}$ interface. For an extreme case that the thickness of the SQW is close to zero, in which $F\left(z, \nu_{0}\right)$ can be treated as a delta function $\delta\left(z, \nu_{0}\right)$, the strain wave form $w(t-z / V)$ can be consequently measured by the modulated optical transmission $\Delta T(t)$. For the real case, the smaller the SQW thickness is, the closer the modulated op- 

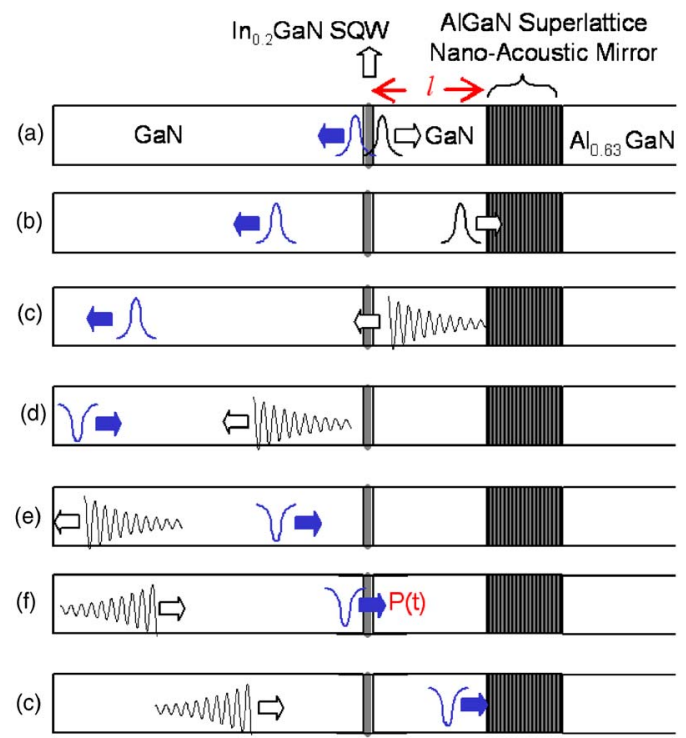

(d)

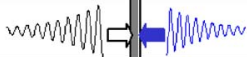

FIG. 1. (Color online) (a) Schematics of the designed acoustic phonon cavity. After the acoustic phonon pulses are generated in the SQW, the propagation of acoustic phonon pulses in the cavity is illustrated through (a) to $(\mathrm{h})$.

tical transmission $\Delta T$ is to the real strain wave form $w(t$ $-z / V)$. With a $7 \mathrm{~nm}$ SQW thickness, corresponding to a traveling time $<1 \mathrm{ps}$ for longitudinal acoustic waves, it enabled us to directly measure strain wave forms in the time domain with a sub terahertz bandwidth.

To study the reflection characteristics of the nanoacoustic superlattice, we designed a phonon cavity as shown in Fig. 1(a). The air/GaN interface is used as an acoustic mirror for totally reflecting acoustic phonon wave packets due to the large acoustic-impedance mismatch. ${ }^{8}$ On the other side, the $\mathrm{AlGaN}$ superlattice is used as the other mirror to selectively reflect acoustic phonon wave packets. Inside the phonon cavity, the SQW is not only an acoustic sensor but also an acoustic generator. By using femtosecond optical pulses, longitudinal acoustic (LA) phonon wave packets can be excited in the SQW primarily through piezoelectric effects ${ }^{10,15}$ and deformation-potential couplings. ${ }^{10,13,15-17}$ After the LA phonon pulses were generated, they left the SQW in counterdirections as shown in Figs. 1(a)-1(h).

To obtain the reflection transfer function of the superlattice mirror, we analyze the strain waves propagating in the phonon cavity in the frequency domain. By taking the Fourier transform of Eq. (1) and letting $t^{\prime}=t-z / V$, we can obtain

$$
\begin{aligned}
\Im\{\Delta T(t)\} & =\int_{-\infty}^{\infty} d z F(z) e^{i(\omega / V) z} \int_{-\infty}^{\infty} d t^{\prime} w\left(t^{\prime}\right) e^{i \omega t^{\prime}} \\
& =F(\omega) W(\omega),
\end{aligned}
$$

where $\mathfrak{I}$ denotes the Fourier transform and $F(\omega)$ and $W(\omega)$ are the Fourier transforms of the SQW sensitivity function and the strain wave, respectively. By tracing the initiated phonon wave packet toward the surface side (marked by the solid arrow) as shown in Figs. 1(a)-1(f), we denote $W_{P}(\omega)$ as the strain wave form reflected from the surface and reentering the SQW. The Fourier transform of the measured temporal signal through the SQW $P(t)$ [as shown in Fig. 1(f)] can be related to the acoustic response of the $\mathrm{SQW} F(\omega)$ as phonons in the GaN-based cavity. The oscillation frequency
Downloaded 16 Feb 2009 to 140.112.113.225. Redistribution subject to AlP license or copyright; see http://apl.aip.org/apl/copyright.jsp

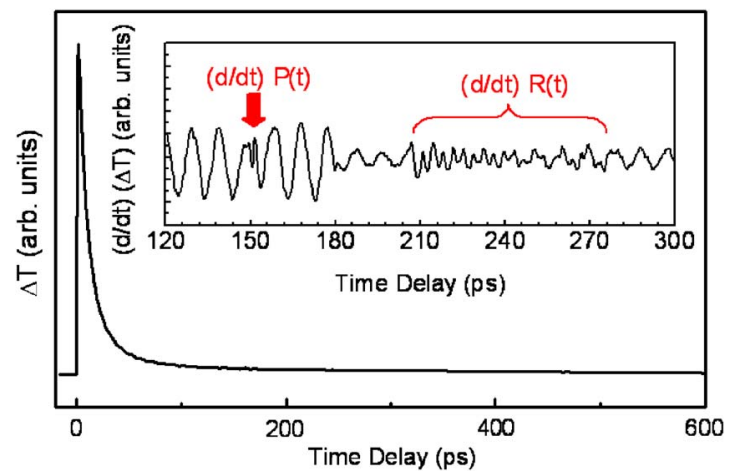

FIG. 2. (Color online) Measured transmission changes of the optical probe pulse as a function of time delay. The differentiated result is shown in the inset.

$$
\Im\{P(t)\}=W_{P}(\omega) F(\omega) .
$$

Please note that the pulse width of the LA phonon generated in the $7 \mathrm{~nm} \mathrm{SQW}$ is on the order of $1 \mathrm{ps}$ (corresponding to $1 \mathrm{THz}$ ) since the longitudinal acoustic velocity is $\sim 8 \mathrm{~nm} / \mathrm{ps}$. However, $W_{P}(\omega)$ may not be the same as the original strain wave form generated in the SQW due to frequencydependent propagation loss and the air/GaN interface roughness.

If we further trace the coherent acoustic phonons as shown in Figs. 1(f) $-1(\mathrm{~h})$, the strain wave form $W_{P}(\omega)$ propagates for a distance $l$, is reflected by the superlattice mirror, and propagates back to the SQW. The strain wave form for one round-trip is $W_{R}(\omega)=W_{P}(\omega) R_{\mathrm{SL}}(\omega) A(\omega, 2 l)$, where $R_{\mathrm{SL}}(\omega)$ is the reflection transfer function of the superlattice mirror and $A(\omega, 2 l)$ is the propagation loss for the distance $2 l$. Similarly, the Fourier transform of the measured temporal signal $R(t)$ [corresponding to Fig. 1(h)] can be expressed as

$$
\Im\{R(t)\}=2 W_{R}(\omega) F(\omega),
$$

where the factor 2 results from the propagation of acoustic phonons in counterdirections. Combining Eqs. (3) and (4), we can thus experimentally obtain the reflection transfer function of the superlattice mirror, $R_{\mathrm{SL}}(\omega)$, by comparing $R(t)$ and $P(t)$ with

$$
R_{\mathrm{SL}}(\omega) \propto \frac{\Im\{R(t)\}}{\Im\{P(t)\}}\left(\frac{1}{A(\omega, 2 l)}\right) .
$$

The 17 -period $8 \mathrm{~nm} / 8 \mathrm{~nm} \mathrm{Al} \mathrm{l}_{0.7} \mathrm{Ga}_{0.3} \mathrm{~N} / \mathrm{Al}_{0.2} \mathrm{Ga}_{0.8} \mathrm{~N}$ superlattice was grown on a $1.2-\mu$ m-thick $\mathrm{Al}_{0.63} \mathrm{Ga}_{0.37} \mathrm{~N}$ buffer layer on a $c$-plane sapphire substrate. ${ }^{18}$ On top of the superlattice mirror, a 7-nm width $\operatorname{In}_{0.2} \mathrm{Ga}_{0.8} \mathrm{~N}$ was sandwiched between a 240-nm-thick GaN buffer layer and a 600-nm-thick GaN cap layer. All the nitride-based materials had a hexagonal structure and a growth direction of (0001). The experiments were performed by a degenerate femtosecond pumpprobe system at room temperature. The photon energy was $3.1 \mathrm{eV}$, which was below the band gaps of $\mathrm{GaN}(\sim 3.4 \mathrm{eV})$ and each $\mathrm{AlGaN}(>3.4 \mathrm{eV})$ layer but higher than that of $\mathrm{In}_{0.2} \mathrm{GaN} \mathrm{SQW}(\sim 2.8 \mathrm{eV})$.

Figure 2 shows the measured transmission variations as a function of time delay. The trace was differentiated as shown in the inset of Fig. 2 for easier observation of the oscillations. A background $107 \mathrm{GHz}$ oscillation signal was observed, which is due to the propagation of acoustic phonons in the GaN-based cavity. The oscillation frequency 


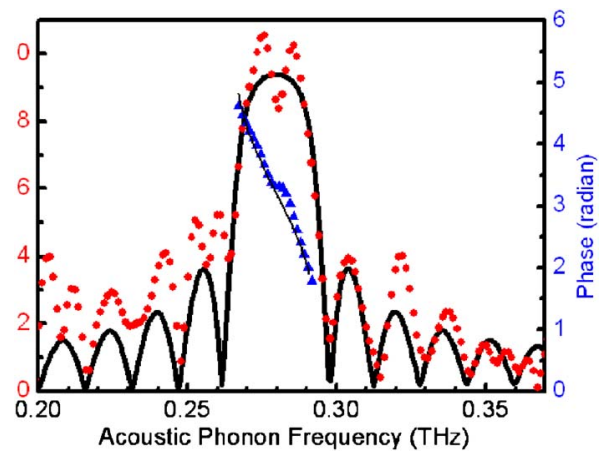

FIG. 3. (Color online) Experimentally obtained amplitude (in dots) and phase (in triangles) spectra of the reflection transfer function of the nanoacoustic mirror with superlattice structure. The theoretical amplitude (in thick solid line) and phase (in thin solid line) spectra are obtained by the transfermatrix method.

is not related to the strain wave form but is determined by the sound velocity of the acoustic phonons and optical properties of the probe pulses. ${ }^{19-21}$ Similar effects were experimentally observed in previous studies. ${ }^{22-24}$ This background $107 \mathrm{GHz}$ signal does not interfere with our analysis since the frequency range of our interest is near the first phononic band gap of the superlattice.

When the LA phonon waves traveled through the SQW, the transmitted optical probe power was dominantly modulated in this region. As shown in the inset of Fig. 2, a oneperiod sine wave was observed at $151 \mathrm{ps,} \mathrm{corresponding} \mathrm{to}$ the differentiated signal of $P(t)$ as expected in Fig. 1(f). Although the trace appears as a one-period sinelike wave form after differentiation, the undifferentiated wave form was actually a negative Gaussian-like pulse. Starting at 209 ps, a $0.28 \mathrm{THz}$ oscillation can be clearly resolved, which is the differentiated signal of the round-trip wave form $R(t)$. From Eq. (5), the reflection transfer function is determined not only by the term $\mathfrak{I}\{R(t)\} / \mathfrak{I}\{P(t)\}$ but also by the propagation loss function $A(\omega, 2 l)$, which is frequency dependent. ${ }^{25}$ To estimate the propagation loss in GaN, we compared the measured wave oscillations after propagating one and two roundtrips inside the phonon cavity. By considering the calculated reflectivity of the phonon mirrors, the acoustic attenuation coefficient could be estimated to be $\sim 0.5 \mu \mathrm{m}^{-1}$ at $0.28 \mathrm{THz}$. If one assumed that the propagation loss is $\omega^{2}$ dependent, ${ }^{25}$ the variation of $A(\omega, 2 l)$ in the frequency range of $0.26-0.30 \mathrm{THz}$ was less than $3.5 \%$, and $A(\omega, 2 l)$ in Eq. (5) could be approximated as a constant near $0.28 \mathrm{THz}$. We can thus obtain the experimentally recovered amplitude (in dots) and phase (in triangles) spectra of $R_{\mathrm{SL}}(\omega)$, the reflection transfer function of the superlattice, as shown in Fig. 3. The reflectivity is higher near $0.28 \mathrm{THz}$, which is close to the theoretical central frequency $(0.279 \mathrm{THz})$ of the first phononic band gap of the superlattice. From the definition of full width at half maximum, the bandwidth of the reflection spectrum is $\sim 30 \mathrm{GHz}$ (which is from 0.265 to $0.295 \mathrm{THz}$ ).

By the transfer-matrix method, ${ }^{3}$ we calculated the reflectivity (in thick solid line) and the phase (in thin solid line) spectra inside the first band gap of the superlattice mirror, as shown in Fig. 3. The mass densities we used for $\mathrm{GaN}$, $\mathrm{Al}_{0.7} \mathrm{Ga}_{0.3} \mathrm{~N}, \mathrm{Al}_{0.2} \mathrm{Ga}_{0.7} \mathrm{~N}$, and $\mathrm{Al}_{0.63} \mathrm{Ga}_{0.27} \mathrm{~N}$ were 6150, 4340, 5332 , and $4515 \mathrm{~kg} / \mathrm{m}^{3}$, respectively. The stiffness coefficients $C_{33}$ were $382,388.2,384.8$, and $387.6 \mathrm{GPa}$. Compared with the calculated results, it is interesting that there is a small dip in the experimentally retrieved amplitude spectrum as well as a plateau in the corresponding phase spectrum at $\sim 0.28 \mathrm{THz}$. This dip could be attributed to defects in the superlattice or a measurement artifact. Overall the calculated results are in agreement with the experimentally recovered transfer function of the nanoacoustic superlattice around the first phononic band gap. Although the transfer-matrix method is based on elasticity continuum with the assumption that the wavelength is much longer than the atom spacing, our study indicates that both phase and amplitude spectra of the nanoacoustic superlattice can still be explained well by the transfer-matrix method.

In summary, we have studied the characteristics of a nanoacoustic mirror and a nanophononic cavity in the time domain by using a piezoelectric SQW. The phase-resolved transfer function of the nanoacoustic superlattice and the properties of the nanophononic cavity have been experimentally obtained and investigated.

The authors acknowledge G.-W. Chern, G.-Y. Guo, and A.-T. Tien for stimulating scientific discussions. This work was sponsored by the National Science Council of Taiwan under Grant No. 95-2120-M-002-013.

${ }^{1}$ M. S. Kushwaha, P. Halevi, L. Dobrzynski, and B. Djafarirouhani, Phys. Rev. Lett. 71, 2022 (1993).

${ }^{2}$ C. Colvard, R. Merlin, M. V. Klein, and A. C. Gossard, Phys. Rev. Lett. 45, 298 (1980).

${ }^{3}$ S. Mizuno and S. Tamura, Phys. Rev. B 45, 734 (1992).

${ }^{4}$ V. Narayanamurti, H. L. Stormer, M. A. Chin, A. C. Gossard, and W. Wiegmann, Phys. Rev. Lett. 43, 2012 (1979).

${ }^{5}$ N. M. Stanton, R. N. Kini, A. J. Kent, M. Henini, and D. Lehmann, Phys. Rev. B 68, 113302 (2003).

${ }^{6}$ M. Trigo, A. Bruchhausen, A. Fainstein, B. Jusserand, and V. ThierryMieg, Phys. Rev. Lett. 89, 227402 (2002).

${ }^{7}$ K.-H. Lin, G.-W. Chern, Y.-K. Huang, and C.-K. Sun, Phys. Rev. B 70, 073307 (2004)

${ }^{8}$ K.-H. Lin, C.-T. Yu, S.-Z. Sun, H.-P. Chen, C.-C. Pan, J.-I. Chyi, S.-W. Huang, P.-C. Li, and C.-K. Sun, Appl. Phys. Lett. 89, 043106 (2006).

${ }^{9}$ C.-L. Hsieh, K.-H. Lin, S.-B. Wu, C.-C. Pan, J.-I. Chyi, and C.-K. Sun, Appl. Phys. Lett. 85, 4735 (2004).

${ }^{10}$ C.-T. Yu, K.-H. Lin, C.-L. Hsieh, C.-C. Pan, J.-I. Chyi, and C.-K. Sun, Appl. Phys. Lett. 87, 093114 (2005).

${ }^{11}$ K.-H. Lin, G.-W. Chern, C.-T. Yu, T.-M. Liu, C.-C. Pan, G.-T. Chen, J.-I. Chyi, S.-W. Huang, P.-C. Li, and C.-K. Sun, IEEE Trans. Ultrason. Ferroelectr. Freq. Control 52, 1404 (2005).

${ }^{12}$ N. D. Lanzillotti-Kimura, A. Fainstein, A. Lemaitre, and B. Jusserand, Appl. Phys. Lett. 88, 083113 (2006).

${ }^{13}$ G.-W. Chern, C.-K. Sun, G. D. Sanders, and C. J. Stanton, Top. Appl. Phys. 92, 339 (2004).

${ }^{14}$ G.-W. Chern, K.-H. Lin, and C.-K. Sun, J. Appl. Phys. 95, 1114 (2004).

${ }^{15}$ C.-K. Sun, J.-C. Liang, and X.-Y. Yu, Phys. Rev. Lett. 84, 179 (2000).

${ }^{16}$ J. J. Baumberg, D. A. Williams, and K. Kohler, Phys. Rev. Lett. 78, 3358 (1997).

${ }^{17}$ O. Matsuda, T. Tachizaki, T. Fukui, J. J. Baumberg, and O. B. Wright, Phys. Rev. B 71, 115330 (2005).

${ }^{18}$ S. Keller, P. Waltereit, P. Cantu, U. K. Mishra, J. S. Speck, and S. P. DenBaars, Opt. Mater. (Amsterdam, Neth.) 23, 187 (2003).

${ }^{19}$ H. T. Grahn, H. J. Maris, and J. Tauc, IEEE J. Quantum Electron. 25, 2562 (1989).

${ }^{20}$ O. Matsuda and O. B. Wright, J. Opt. Soc. Am. B 19, 3028 (2002).

${ }^{21}$ R. L. Liu, G. D. Sanders, C. J. Stanton, C. S. Kim, J. S. Yahng, Y. D. Jho, K. J. Yee, E. Oh, and D. S. Kim, Phys. Rev. B 72, 195335 (2005).

${ }^{22}$ K.-H. Lin, C.-T. Yu, Y.-C. Wen, and C.-K. Sun, Appl. Phys. Lett. 86, 093110 (2005)

${ }^{23}$ J. S. Yahng, Y. D. Jho, K. J. Yee, E. Oh, J. C. Woo, D. S. Kim, G. D. Sanders, and C. J. Stanton, Appl. Phys. Lett. 80, 4723 (2002).

${ }^{24}$ Y.-K. Huang, G.-W. Chern, C.-K. Sun, Y. Smorchkova, S. Keller, U. Mishra, and S. P. DenBaars, Appl. Phys. Lett. 79, 3361 (2001).

${ }^{25}$ T. C. Zhu, H. J. Maris, and J. Tauc, Phys. Rev. B 44, 4281 (1991). 